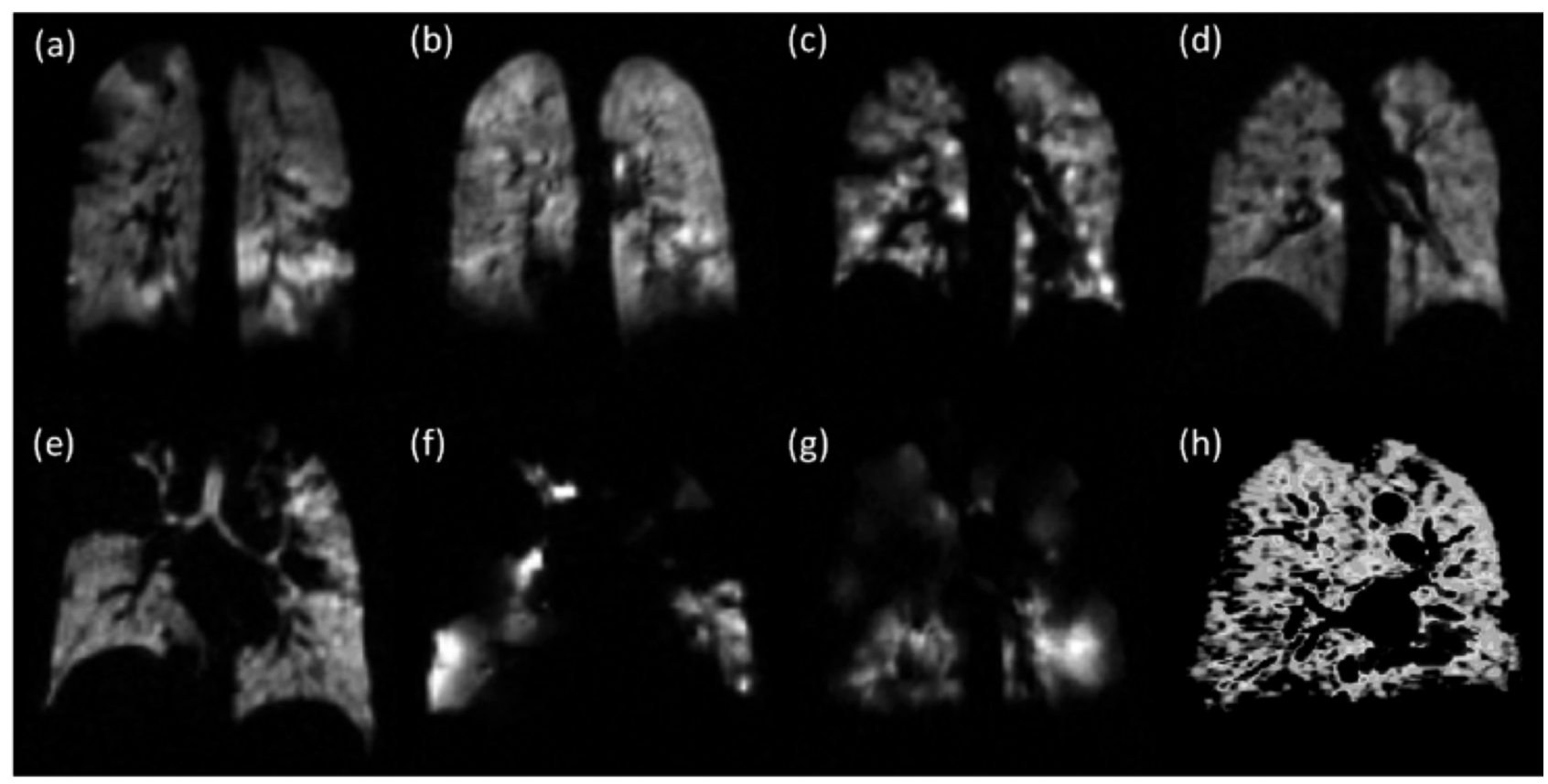

Abstract P283 Figure 1 Example of ${ }^{3} \mathrm{He}$ ventilation images; non-CF bronchiectasis patient (a) before and (b) after IV anti biotics, asthma patient (c) before and (d) after bronchodilator, (e) CF patient, and (f) COPD patient I a ${ }^{129}$ Xe ventilation image (g) COPD patient 2, and a ${ }^{1} \mathrm{H}$ perfusion image (h) patient with poor gas transfer

complex asthma (scanned before and after bronchodilator inhalation), CF, patients with poor gas transfer but well-preserved lung parenchyma on CT, IPF overlapping with emphysema.

Results Figure 1 shows example images from a cross-section of patients scanned, details of the individual cases will be expanded upon. No adverse events related to imaging were reported. In terms of imaging workflow, scan time average was between 30 min and $1 \mathrm{~h} 30 \mathrm{~min}$. Patients have been referred from clinics within a $100 \mathrm{~km}$ radius and we are also active in enabling novice sites further afield with the technology.

Conclusion Hyperpolarised gas MR provides sensitive, regional images of lung function which can be used to aid in clinical decision making on an individual patient basis. With improvements in gas polarisation, MR hardware and image acquisition techniques routine clinical lung imaging with the cheaper gas isotope ${ }^{129} \mathrm{Xe}$ is also now possible and large scale clinical evaluation of these methods in patient populations are now underway as part of clinical work up.

\section{P284 V/Q SCANNING USING OXYGEN-ENHANCED MAGNETIC RESONANCE IMAGING}

JL Ulloa, AR Morgan, T Lacey, C Roberts, GJM Parker. Bioxydyn Limited, Manchester, UK

10.1136/thoraxjnl-2015-207770.420

Ventilation-perfusion (V/Q) scan is an established technique used to monitor lung dysfunction in conditions such as pulmonary embolism. ${ }^{1}$ However, there are safety concerns regarding its use in several important populations e.g. pregnant women and children. Recent advances have transformed the use of standard clinical MRI equipment in functional lung imaging. ${ }^{2}$
We recently presented a clinically appropriate dynamic oxygenenhanced MRI (dOE-MRI) protocol. ${ }^{3}$ In this work, we interpret dOE-MRI data with a novel physiological model of gas exchange $^{4}$ that maps the regional distribution of lung ventilation and perfusion, simulating results from a standard V/Q scan.

3D dOE-MRI data were acquired using a 1.5 T Philips scanner as in: ${ }^{3}$ a 5 min baseline $\mathrm{T}_{1}$ acquisition, followed by a $15 \mathrm{~min}$ dynamic acquisition, during which the subject breathed 100\% $\mathrm{O}_{2}$ for the middle $5 \mathrm{~min}$. Total scan time is under $30 \mathrm{~min}$ throughout which the subject breathes normally. The partial pressure of oxygen in the alveolar compartment was derived as proposed in ${ }^{5}$ and maps of regional alveolar ventilation and perfusion were produced using a novel two-compartmental model of gas exchange based on. ${ }^{4}$

Figure 1 shows an example of whole-chest ventilation and perfusion maps from a healthy subject overlaid on a single volume of the acquired MRI (left column). The same maps are displayed as anterior/posterior and right/left anterior oblique projections (right column), equivalent to those achieved with V/ Q scintigraphy.

Estimation of alveolar ventilation and perfusion distribution from 3D dynamic OE-MRI is feasible and can be obtained under clinically acceptable conditions using standard radiological equipment without ionising radiation risks. This approach opens the possibility for regional assessment of lung physiology using a single short scanning session.

\section{REFERENCES}

1 Bajc, et al. Eur I Nucl Med Mol Imaging 2009;36(8):1356-70

2 Wild, et al. Insights Imaging 2012;3(4):345-53

3 Ulloa, et al. Proc Intl Soc Mag Reson Med. 2015:23:3973

4 Naish, et al. Proc Intl Soc Mag Reson Med. 2010;18:2516

5 Kershaw, et al. MRM 2010;64:1838-42 